\title{
Algebraic values of Schwarz triangle functions
}

\author{
Hironori Shiga \\ Inst. of Math. and Physics \\ Chiba University \\ Yayoi-cho 1-33, Inage-ku \\ Chiba 263-8522, Japan \\ shiga@math.s.chiba-u.ac.jp
}

\author{
Jürgen Wolfart \\ Math. Sem. der Univ. \\ Postfach 111932 \\ D-60054 Frankfurt a.M., \\ Germany \\ wolfart@math.uni-frankfurt.de
}

27th May 2005

\begin{abstract}
We consider Schwarz maps for triangles whose angles are rather general rational multiples of $\pi$. Under which conditions can they have algebraic values at algebraic arguments? The answer is based mainly on considerations of complex multiplication of certain Prym varieties in Jacobians of hypergeometric curves. The paper can serve as an introduction to transcendence techniques for hypergeometric functions, but contains also new results and examples.
\end{abstract}

MSC classification: Primary 14K22, Secondary 30C20, 33C05, 11J91, 11.J95

Keywords: Schwarz triangle functions, hypergeometric functions, algebraic values, transcendence, complex multiplication

Hypergeometric functions have many interesting relations to arithmetics, for example to modular forms, diophantine approximation, continued fractions and so on. In the following contribution we will concentrate on transcendence questions and explain the relevant techniques in the framework of a question concerning the classical Schwarz triangle functions.

These triangle functions $D\left(\nu_{0}, \nu_{1}, \nu_{\infty} ; z\right)$ are defined as quotients of two linearly independent solutions of Gauss' hypergeometric differential equations. If their angular parameters $\nu_{0}, \nu_{1}, \nu_{\infty}$ are real and have absolute value in the open interval $] 0,1[$, they define biholomorphic mappings of the complex upper half plane $\mathcal{H}$ onto triangles in the Riemann sphere bounded by circular arcs. The singular points $0,1, \infty$ of the differential equation are sent by $D$ to the vertices of the triangle including there angles $\pi\left|\nu_{0}\right|, \pi\left|\nu_{1}\right|, \pi\left|\nu_{\infty}\right|$, 
respectively. Particularly interesting special cases are those where $\nu_{0}, \nu_{1}, \nu_{\infty}$ are the inverses of positive integers $p, q, r$ because then $D$ is the inverse function of an automorphic function for the triangle group with signature $\langle p, q, r\rangle$, isomorphic to the (projective) monodromy group of the hypergeometric differential equation.

The present paper considers the question if Schwarz triangle functions can have algebraic values at algebraic arguments. The problem has its origins in the natural general question if or under which conditions (suitably normalized) transcendental functions have transcendental values at algebraic arguments, and in this special context it is related to automorphic functions and periods of abelian varieties. For a general survey about algebraic and transcendental periods in number theory see Waldschmidt's recent article [20]. In the cases related to automorphic functions mentioned above the problem is treated already in our previous paper [16, Cor. 5]. It turned out that a positive answer is directly related to the condition if certain Prym varieties are of complex multiplication (CM) type, the Pryms being defined in a natural way via the integral representation of the associated hypergeometric functions. Now we generalize the setting and consider arbitrary rational angular parameters $\nu_{0}, \nu_{1}, \nu_{\infty}$, restricted only by some mild technical condition excluding logarithmic singularities and some other very special situations. The main results will show that we have still 'CM' as necessary condition for 'algebraic values at algebraic arguments', but that even under the CM condition this algebraicity is rather exceptional. However, we will give examples that such exceptions occur.

This more general type of triangle functions has still images of $\mathcal{H}$ bounded by parts of circles but they are in general not globally biholomorphic - the image domains may overlap with themselves. We treated in [15] an analogous problem admitting apparent singularities in the associate Fuchsian differential equations. In many cases, the triangle functions of the present paper may in fact be considered as limit cases of those of [15], and many techniques developed there are useful also for the problem treated in the present paper. Therefore we collect in Section 1 some known material mainly from [15], [16], [21]. Section 2 presents the necessary tools from transcendence theory, and in Section 3 we state and prove the main results. The methods rely in part on the classical theory of hypergeometric functions, in part on the consideration of families of abelian varieties, and in part on Wüstholz' transcendence techniques [24]. Sections 4 and 5 present instructive examples.

Notation: we will call Propositions the statements we took from the literature and Theorems the new results presented here even if they might be less important than the Propositions. 


\section{Families of Prym varieties and associate func- tions}

\subsection{Integral representation by the periods on curves}

Throughout this paper we will suppose that the angular parameters satisfy

$$
\nu_{0}, \nu_{1}, \nu_{\infty} \in \mathbb{Q}-\mathbb{Z}, \quad \nu_{0} \pm \nu_{1} \pm \nu_{\infty} \notin \mathbb{Z}
$$

We will use the integral representation of the Gauss hypergeometric function $F(a, b, c ; z)$ - omitting the usual normalizing Beta factor and some algebraic nonzero factors, see Section 5 of [15] for a careful discussion - in the form

$\int_{\gamma} u^{a-c}(u-1)^{c-b-1}(u-z)^{-a} d u=\int_{\gamma} u^{-\mu_{0}}(u-1)^{-\mu_{1}}(u-z)^{-\mu_{z}} d u=\int_{\gamma} \eta(z)$

with the (rational) exponents

$$
\begin{array}{r}
\mu_{0}=\frac{1}{2}\left(1-\nu_{0}+\nu_{1}-\nu_{\infty}\right) \\
\mu_{1}=\frac{1}{2}\left(1+\nu_{0}-\nu_{1}-\nu_{\infty}\right) \\
\mu_{z}=\frac{1}{2}\left(1-\nu_{0}-\nu_{1}+\nu_{\infty}\right) \\
\mu_{\infty}=\frac{1}{2}\left(1+\nu_{0}+\nu_{1}+\nu_{\infty}\right) \\
\mu_{0}+\mu_{1}+\mu_{z}+\mu_{\infty}=2
\end{array}
$$

for some Pochhammer cycle $\gamma$ around two of the singularities $0,1, z, \infty$. As already remarked by Klein [10, §19], analytic continuation of $F(a, b, c ; z)$ means only to replace $\gamma$ by another cycle of integration, and a basis of solutions of the corresponding hypergeometric differential equation will be obtained by taking two Pochhammer cycles around different pairs of singularities: remark that our hypothesis on the sums of the angular parameters guarantees that no exponent $\mu_{j}$ is an integer, whence all singularities are nontrivial. For fixed arguments $z \neq 0,1, \infty$ this integral representation can be seen as a period integral on a nonsingular projective model $X(k, z)$ of the algebraic curve

$$
y^{k}=u^{k \mu_{0}}(u-1)^{k \mu_{1}}(u-z)^{k \mu_{z}}
$$


where $k$ is the least common denominator of the $\mu_{j}, \gamma$ some homology cycle on $X(k, z)$, and $\eta$ a differential given on the singular model as

$$
\eta=\eta(z)=\frac{d u}{y} .
$$

It is a second kind differential what can be seen using appropriate local variables ([21]; N. Archinard [1] explains in more detail the desingularization procedure). Our Schwarz triangle map is a multivalued analytic function on $\mathbb{C}-\{0,1\}$ defined by

$$
D\left(\nu_{0}, \nu_{1}, \nu_{\infty} ; z\right)=D(\eta ; z)=D(z)=\frac{\int_{\gamma_{1}} \eta(z)}{\int_{\gamma_{2}} \eta(z)}
$$

for some independent cycles $\gamma_{1}, \gamma_{2}$ on $X(k, z)$.

In the next subsection we will give a precise definition of independence for these cycles, for the moment we can assume that they come from Pochhammer cycles around different pairs of singularities and are locally independent of $z \neq 0,1, \infty$. The triangle functions extend continuously to the arguments excluded here, and our normalization guarantees that $D(0), D(1), D(\infty)$ become algebraic or $\infty$, see [15, Section 3.1]. For later use recall the relation between angular and exponential parameters and $a, b, c$.

$$
\begin{gathered}
\nu_{0}=1-c=1-\mu_{0}-\mu_{z}=\mu_{1}+\mu_{\infty}-1 \\
\nu_{1}=c-a-b=1-\mu_{1}-\mu_{z}=\mu_{0}+\mu_{\infty}-1 \\
\nu_{\infty}=a-b=\mu_{z}+\mu_{\infty}-1=1-\mu_{0}-\mu_{1} .
\end{gathered}
$$

\subsection{The family of Prym varieties}

The family of Prym varieties in question can be described as follows. For all proper divisors $d$ of $k$ there is an obvious morphism of the curve $X(k, z)$ onto the curve $X(d, z)$ in whose definition (1.2) we keep fixed the exponential parameters $k \mu_{i}$ on the right hand side and replace $k$ by $d$ as exponent of $y$. These morphisms induce epimorphisms

$$
\operatorname{Jac} X(k, z) \rightarrow \operatorname{Jac} X(d, z) .
$$

Let $T(k, z)$ be the connected component of 0 in the intersection of all kernels of these epimorphisms. Then it is known by [21], [1] that $T(k, z)$ is an abelian variety of dimension $\varphi(k)$ where $\varphi$ denotes Euler's function. $T(k, z)$ has a special endomorphism structure called generalized complex 
multiplication (complex multiplication in the narrow sense will be treated in Subsection 2.3) by the cyclotomic field

$$
\mathbb{Q}\left(\zeta_{k}\right) \subseteq \text { End }_{0} T(k, z):=\mathbb{Q} \otimes_{\mathbb{Z}} \text { End } T(k, z)
$$

induced by an automorphism of the curve $X(k, z)$ described on its singular model by

$$
\sigma:(u, y) \mapsto\left(u, \zeta_{k}^{-1} y\right), \quad \zeta_{k}=e^{\frac{2 \pi i}{k}} .
$$

If $\langle s\rangle$ denotes the fractional part $s-[s]$ of $s \in \mathbb{Q}$, the CM type of $T(k, z)$ can be easily calculated in terms of the $\mu_{j}$ by

$$
r_{n}=\operatorname{dim} W_{n}=-1+\sum_{j}\left\langle\mu_{j} n\right\rangle,
$$

where $W_{n}$ denotes the eigenspace for the eigenvalue $\zeta_{k}^{n}$ for the action of $\sigma$ on the vector space $H^{0}(T(k, z), \Omega)$ of the first kind differentials, see e.g. [16] (on p.23 use formula (4) with $N=2$ ) or [3]. Note that $r_{n}$ can take the values $0,1,2$ only and satisfies $r_{n}+r_{-n}=2$ for all $n$.

In the following we will consider the second kind differentials $\eta$ always as differentials on $T(k, z)$ and the cycles $\gamma_{1}, \gamma_{2}$ as cycles of the homology in $T(k, z)$. This homology $H_{1}(T(k, z), \mathbb{Z})$ is a $\mathbb{Z}\left[\zeta_{k}\right]$-module of rank two, and independence of the cycles in the definition of the normalized Schwarz triangle function $D(z)=\int_{\gamma_{1}} \eta(z) / \int_{\gamma_{2}} \eta(z)$ means now $\mathbb{Q}\left(\zeta_{k}\right)$-linear independence in the $\mathbb{Q}\left(\zeta_{k}\right)$-module $H_{1}(T(k, z), \mathbb{Q})=\mathbb{Q} \otimes_{\mathbb{Z}} H_{1}(T(k, z), \mathbb{Z})$. Note that for algebraic $z$ the curve, its Jacobian, its Prym variety $T(k, z)$ and the differential $\eta(z)$ are all defined over number fields.

\subsection{Associate functions}

As common in the literature about hypergeometric functions, we call two hypergeometric functions $F(a, b, c ; z), F\left(a^{\prime}, b^{\prime}, c^{\prime} ; z\right)$ associate if

$$
a \equiv a^{\prime}, b \equiv b^{\prime}, c \equiv c^{\prime} \bmod \mathbb{Z}
$$

or equivalently, if the respective angular parameters satisfy

$$
\begin{array}{r}
\nu_{0} \equiv \nu_{0}^{\prime}, \nu_{1} \equiv \nu_{1}^{\prime}, \nu_{\infty} \equiv \nu_{\infty}^{\prime} \bmod \mathbb{Z} \quad \text { and } \\
\nu_{0}+\nu_{1}+\nu_{\infty} \equiv \nu_{0}^{\prime}+\nu_{1}^{\prime}+\nu_{\infty}^{\prime} \bmod 2 \mathbb{Z}
\end{array}
$$

or if the respective exponential parameters satisfy

$$
\mu_{j} \equiv \mu_{j}^{\prime} \bmod \mathbb{Z} \quad \text { for all } \quad j=0,1, z, \infty \quad \text { and } \quad \sum_{j} \mu_{j}=\sum_{j} \mu_{j}^{\prime}=2 .
$$


All functions associate to $F(a, b, c ; z)$ generate a vector space of dimension two over the field of rational functions $\mathbb{C}(z)$, and since our parameters are supposed to be rational, between any three associate functions there is a linear relation with coefficients in $\mathbb{Q}(z)$. These relations can explicitely produced by means of Gauss' relations between contiguous functions, see [8]. Any two associate hypergeometric functions generate the vector space over $\mathbb{C}(z)$ (obvious exceptions like $F(a, a+1, c ; z), F(a+1, a, c ; z)$ are excluded by our assumptions about the angular parameters). The congruences for the exponential parameters imply that the differentials $\eta, \eta^{\prime}$ differ only by factors which are rational functions $R(u, z) \in \mathbb{Q}(u, z)$. As second kind differentials on the Prym variety $T(k, z)$ they belong therefore to the same $\mathbb{Q}\left(\zeta_{k}\right)$-eigenspace $V_{1}$ in its de Rham cohomology. In our normalization, the differentials of this eigenspace are characterized by $\eta \circ \sigma=\zeta_{k} \eta$. The intersection of $V_{1}$ with $H^{0}(T(k, z), \Omega)$ gives the eigenspace $W_{1}$ mentioned in the definition of the CM type. This observation extends to the other eigenspaces $V_{n}, n \in(\mathbb{Z} / k \mathbb{Z})^{*}$, and the fact that all associate hypergeometric functions generate a 2 -dimensional vector space over $\mathbb{C}(z)$ has an obvious interpretation for the eigenspaces $V_{n}$ in the de Rham cohomology:

Lemma $1.1 \operatorname{dim} V_{n}=2$ for all $n \in(\mathbb{Z} / k \mathbb{Z})^{*}$.

Dimension means here the dimension over $\mathbb{C}$, but for algebraic $z$ we can give another useful interpretation: as already mentioned, $T(k, z)$ is then defined over $\overline{\mathbb{Q}}$, all differentials $\eta$ in the integral representation are defined over $\overline{\mathbb{Q}}$ as well whence we consider the vector spaces

$$
H^{0}(T(k, z), \Omega), H_{D R}^{1}(T(k, z)), V_{n}
$$

of differentials of the first and second kind defined over $\overline{\mathbb{Q}}$ as vector spaces over $\overline{\mathbb{Q}}$. In this sense, the Lemma remains true as a statement about $\overline{\mathbb{Q}}-$ dimensions. For $z \in \overline{\mathbb{Q}}$ we will follow this interpretation.

In the proof of Lemma 1.1 there is only one point which is not obvious: even if associate differentials $\eta(z)$ generate a 2 -dimensional $\mathbb{C}(z)$-vector space modulo exact differentials, it could be possible that for some fixed value $z=$ $\tau$ the $\mathbb{C}$-dimension would be smaller if e.g. all differentials in question vanish for $z=\tau$. This breakdown of the dimension can be seen to be impossible for $\tau \neq 0,1, \infty$ either by a careful analysis of the possible relations between contiguous functions or by the fact that the genus of $X(k, z)$ is the same for all $z \neq 0,1, \infty$, hence also $\operatorname{dim} H_{D R}(X(k, z))$ is independent of $z$, see [9, Ch. 3.5]. 


\subsection{Shimura varieties, monodromy groups, and modular groups}

In general, our Prym varieties $T(k, z)$ are only special cases of principally polarized complex abelian varieties $A$ of dimension $\varphi(k)$, with period lattice isomorphic to $\mathbb{Z}\left[\zeta_{k}\right]^{2}$, and with an action of $\mathbb{Q}\left(\zeta_{k}\right) \subseteq$ End $_{0} A$ of (general-

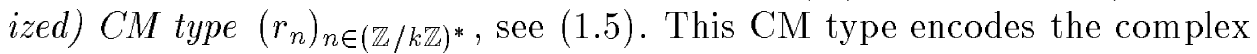
representation of $\mathbb{Q}\left(\zeta_{k}\right)$ on the space of holomorphic differentials $H^{0}(A, \Omega)$ such that $r_{n}$ is the dimension of the eigenspace on which $\zeta_{k}$ acts via

$$
\zeta_{k}: \omega \mapsto \zeta_{k}^{n} \cdot \omega
$$

If we denote the family of all these abelian varieties by $\mathcal{A}$, we know by work of Shimura [17] and Siegel [18]

Proposition 1.2 The family $\mathcal{A}$ is parametrized by the product $\mathcal{H}^{r}$ of upper half planes $\mathcal{H}$ with dimension

$$
r=\frac{1}{2} \sum_{n \in(\mathbb{Z} / k \mathbb{Z})^{*}} r_{n} r_{-n} .
$$

Since $0 \leq r_{n} \leq 2$ and $r_{n}+r_{-n}=2$ for all $n$, we may rephrase this statement by saying that the dimension $r$ is half of the number of the one-dimensional $\mathbb{Q}\left(\zeta_{k}\right)$-eigenspaces in $H^{0}(A, \Omega)$. For the special case of the Prym varieties $A=T(k, z)$ in question, we may take generators $\omega_{j}, j=1, \ldots, r$ of one-dimensional eigenspaces $W_{n} \subset H^{0}(T(k, z), \Omega), n \in$ $(\mathbb{Z} / k \mathbb{Z})^{*} /\{ \pm 1\}$. Then - up to linear fractional transformations - the values of the triangle functions $D\left(\omega_{j} ; z\right)$ defined by period quotients in (1.3) serve as coordinates of the point in $\mathcal{H}^{r}$ corresponding to $T(k, z)$.

Two points in $\mathcal{H}^{r}$ correspond to isomorphic abelian varieties in $\mathcal{A}$ if and only if they belong to one $\Gamma$-orbit where $\Gamma$ denotes the (arithmetically defined) modular group acting discontinuously on $\mathcal{H}^{r}$. The quotient space $\Gamma \backslash \mathcal{H}^{r}$ is therefore a classifying space for $\mathcal{A}$, the Shimura variety of $\mathcal{A}$. In the case $r=1$ we call it a Shimura curve, of course (we neglect many interesting questions about algebraic or arithmetic stucture of these spaces). One subgroup of $\Gamma$ is well known in the context of hypergeometric functions:

Proposition 1.3 Let $\omega_{1}(z)$ be a generator of a one-dimensional $\mathbb{Q}\left(\zeta_{k}\right)$ eigenspace of $H^{0}(T(k, z), \Omega)$ and let $\Delta$ be the (projective) monodromy group of the hypergeometric functions $\int_{\gamma_{1}} \omega_{1}(z), \int_{\gamma_{2}} \omega_{1}(z)$ used in the definition (1.3). Then $\Delta$ has a natural embedding into the modular group $\Gamma$ of $\mathcal{A}$. 
As already explained in Section 1.1, the monodromy group - defined by analytic continuation of the hypergeometric functions - acts on the homology of $X(k, z)$ without changing the curve, hence leaving fixed the isomorphism class of its Jacobian and of the Prym variety. By consequence they embed into the modular group of the family, acting by fractional linear transformations on the coordinates of $\mathcal{H}^{r}$. For a more detailed explanation and a much stronger version of this proposition see [4]; in fact, there is even a holomorphic modular embedding of $\mathcal{H}$ into $\mathcal{H}^{r}$ compatible with the actions of $\Delta$ and $\Gamma$.

One extreme case of Proposition 1.2 will be very useful in Proposition 2.8 below. It can happen that the dimension of the family $\mathcal{A}$ is $r=0$. This is the case if and only if the modular group and a fortiori the monodromy group $\Delta$ is finite. By the classical reasoning of H.A. Schwarz [14], the fact that the hypergeometric functions and their triangle functions have only finitely many branches is equivalent to state that they are algebraic functions: just observe that the elementary symmetric functions of their branches are single-valued meromorphic, hence rational functions, and note that by the hypotheses (1.1) our hypergeometric differential equations are ir reducible. For other arguments in that direction and their generalization to hypergeometric functions in several variables see [5].

The next interesting case is that of Shimura curves, i.e. the case $r=1$. Then the modular group $\Gamma$ and a fortiori the monodromy group $\Delta$ act as arithmetically defined Fuchsian groups. In Section 3 it will become clear why the arithmeticity of $\Delta$ is so important for our question, and Sections 4 and 5 will discuss in great detail one example, i.e. the family of hypergeometric curves (4.2) with angular parameters $\nu_{0}=-\nu_{1}=\nu_{\infty}=1 / 5$ and

$$
k=5, \quad r_{1}=0, r_{2}=r_{3}=1, r_{4}=2, \quad r=1 .
$$

Caution. On the other hand, there are arithmetically defined monodromy groups for which $r>1$. In these cases the $T(k, z)$ belong to some subfamily of Hodge type of $\mathcal{A}$, i.e. to a Shimura subvariety described by a special splitting behaviour of $T(k, z)$ or - equivalently — by the fact that the common endomorphism algebra of all $T(k, z)$ is strictly larger than $\mathbb{Q}\left(\zeta_{k}\right)$. As an example, take

$$
\nu_{0}=\frac{1}{2}, \nu_{1}=\frac{1}{3}, \nu_{\infty}=\frac{1}{10} .
$$

An obvious calculation leads to $k=30$ and $r=2$. We have $W_{ \pm 1}, W_{ \pm 11}$ as one-dimensional eigenspaces in $H^{0}(T(k, z) \Omega)$. The generators of $W_{1}$ and 
$W_{11}$ lead with (1.3) to triangle functions

$$
D\left(\frac{1}{2}, \frac{1}{3}, \frac{1}{10} ; z\right) \text { and } D\left(\frac{1}{2},-\frac{1}{3}, \frac{1}{10} ; z\right)
$$

which are constant multiples of each other, see [21, (16)]. Recall that these triangle functions give the coordinates of the point in $\mathcal{H}^{2}$ corresponding to $T(k, z)$. Therefore these Pryms are parametrized by an upper half plane $\mathcal{H}$ linearly embedded in $\mathcal{H}^{2}$, and a more detailed analysis shows that they split into two factors, both isogenous to the Pryms of the family (4.2); this is not surprising since the monodromy group for the example (1.6) is the triangle group of signature $\langle 2,3,10\rangle$, an index 6 extension of that one in (4.2) of signature $\langle 5,5,5\rangle$.

\section{Tools from transcendence}

\subsection{The analytic subgroup theorem}

The main instrument to obtain transcendence results for hypergeometric functions is Wüstholz' analytic subgroup theorem, see [23] and [24].

Proposition 2.1 Let $G$ be a connected commutative algebraic group defined over $\overline{\mathbb{Q}}$ of dimension $\operatorname{dim} G>r>0$ and

$$
\varphi: \mathbb{C}^{r} \rightarrow G
$$

an analytic homomorphism whose tangential map $d \varphi$ is an homomorphism of $\overline{\mathbb{Q}}$-vectorspaces. If the image contains a nontrivial algebraic point, i.e. if $\varphi\left(\mathbb{C}^{r}\right)(\overline{\mathbb{Q}}) \neq\{0\}$, there is an algebraic subgroup $H \subseteq \varphi\left(\mathbb{C}^{r}\right)$ defined over $\overline{\mathbb{Q}}$ with $\operatorname{dim} H>0$.

The unexperienced reader may wonder why this is a theorem about transcendental numbers. Let us explain it first with a classical example: Let $G$ be the product $\mathbb{C}^{*} \times \mathbb{C}$ of the multiplicative and the additive group of complex numbers and observe that $\{1\} \times \mathbb{C}$ and $\mathbb{C}^{*} \times\{0\}$ are the only nontrivial connected algebraic subgroups of $G$. As analytic homomorphism take the exponential map

$$
\varphi:(z, w) \mapsto\left(e^{z}, w\right)
$$

restricted to the one-dimensional subspace $z=b w$ of the tangent space $\mathbb{C}^{2}$ of $G$. Now suppose there were an algebraic number $a \neq 0,1$ with an 
algebraic $\operatorname{logarithm} b=\log a$. Then $d \varphi$ and our one-dimensional subspace are defined over $\overline{\mathbb{Q}}$ and the $\varphi$-image contains and algebraic point $(a, 1)$. On the other hand, it does not contain any proper algebraic subgroup of $G$ in contradiction to Proposition 2.1. So we obtain the Lindemann-Weierstrass theorem that $e^{b}$ is transcendental for all algebraic $b \neq 0$. With $b=i \pi$ we get the transcendence of $\pi$ as well.

\subsection{Application to periods}

The application of Proposition 2.1 needed for the values of the Schwarz maps is a powerful theorem about linear independence of periods over $\overline{\mathbb{Q}}$ first stated as Theorem 5 of [23]. The proof has been worked out by Paula Cohen in the appendix of [15].

Proposition 2.2 Let $A$ be an abelian variety isogenous over $\overline{\mathbb{Q}}$ to the direct product $A_{1}^{k_{1}} \times \ldots \times A_{N}^{k_{N}}$ of simple, pairwise non-isogenous abelian varieties $A_{\nu}$ defined over $\overline{\mathbb{Q}}$, with $A_{\nu}$ of dimension $n_{\nu}, \nu=1, \ldots, N$. Then the $\overline{\mathbb{Q}}-$ vector space $\widehat{V}_{A}$ generated by $1,2 \pi i$ together with all periods of differentials, defined over $\overline{\mathbb{Q}}$, of the first and the second kind on $A$, has dimension

$$
\operatorname{dim}_{\overline{\mathbb{Q}}} \widehat{V}_{A}=2+4 \sum_{\nu=1}^{N} \frac{n_{\nu}^{2}}{\operatorname{dim}_{\mathbb{Q}} E n d_{0} A_{\nu}} .
$$

We will not repeat the proof here. To give an impression how linear independence of periods follows from Wüstholz' analytic subgroup theorem, we will however state and prove a simpler and very special case, see also [22, Satz 1].

Proposition 2.3 Let $A$ be a simple abelian variety defined over $\overline{\mathbb{Q}}$, $\omega_{1}, \ldots, \omega_{n} \in H^{0}(A, \Omega)$ a basis of holomorphic differentials on $A$, also defined over $\overline{\mathbb{Q}}$, and let $\gamma \in H_{1}(A, \mathbb{Z})$ be a nonzero cycle on $A$. Then the periods

$$
\int_{\gamma} \omega_{1}, \ldots, \int_{\gamma} \omega_{n}
$$

are linearly independent over $\overline{\mathbb{Q}}$.

Assume the statement to be wrong. Then there is a linear relation

$$
a_{1} \int_{\gamma} \omega_{1}+\ldots+a_{n} \int_{\gamma} \omega_{n}=0
$$


with algebraic coefficients $a_{i}$ not all $=0$. Consider the exponential map

$$
\varphi: \mathbb{C}^{n} \rightarrow A \cong \mathbb{C}^{n} / \Lambda
$$

where $\Lambda$ denotes the period lattice $\left\{\left(\int_{\delta} \omega_{1}, \ldots, \int_{\delta} \omega_{n}\right) \mid \delta \in H^{1}(A, \mathbb{Z})\right\}$ and restrict $\varphi$ to the $(n-1)$-dimensional subspace $S$ given by

$$
a_{1} z_{1}+\ldots+a_{n} z_{n}=0 .
$$

This subspace $S$ and $d \varphi$ are defined over $\overline{\mathbb{Q}}$. By our assumption, the nonzero vector

$$
v:=\left(\int_{\gamma} \omega_{1}, \ldots, \int_{\gamma} \omega_{n}\right)
$$

belongs to the kernel of $\varphi$ and $\varphi(\mathbb{Q} v)$ consists of torsion points of $A$, hence belongs to $A(\overline{\mathbb{Q}})$. Therefore Proposition 2.1 applies, but $A$ is simple and has no proper algebraic subgroup of positive dimension, contradiction.

\subsection{Complex multiplication}

Proposition 2.2 indicates that the splitting of $T(k, z)$ and the endomorphism algebra of its simple components will be very important for the understanding of linear dependence or independence of periods. An extreme case is the situation that the abelian variety $A$ has complex multiplication or $C M$ in short. This means that there is a number field $K \subseteq$ End $_{0} A$ of the (maximal possible) degree $[K: \mathbb{Q}]=2 \operatorname{dim} A$. For the convenience of the reader, we collect here some facts well known from the literature (see e.g. [13]).

The field $K$ is necessarily a $C M$ field, that is a totally imaginary quadratic extension of some totally real number field $F$ of degree $g=\operatorname{dim} A$. The space $H_{D R}(A)$ of all first and second kind differentials splits into $2 \operatorname{dim} A$ one-dimensional subspaces $V_{\sigma}$ where $\sigma$ runs over all embeddings $K \rightarrow \mathbb{C}$ and every $\alpha \in K$ acts on $V_{\sigma}$ by multiplication with $\sigma(\alpha)$. The subspace $H^{0}(A, \Omega)$ of first kind differentials splits under the action of $K$ into $g$ onedimensional eigenspaces $W_{\sigma}=V_{\sigma}$ among them for which $\sigma$ runs over a system of representatives of all embeddings $K \rightarrow \mathbb{C}$ modulo complex conjugation. (In the case of a cyclotomic field $\mathbb{Q}\left(\zeta_{k}\right)$ we may caracterize the embeddings $\sigma$ as usual by representatives of prime residue classes in $\mathbb{Z} / k \mathbb{Z}$ modulo \pm 1 .) The collection of these representatives $\sigma$ are called the $C M$ type of $A$ and determine $A$ uniquely up to isogeny. The abelian varieties with this endomorphism structure form a zero-dimensional Shimura variety, and $A$ is defined over a (particularly interesting!) number field. 
It can happen that $A$ with $\mathrm{CM}$ is not simple: it may be isogeneous to some power $B^{m}$ of a simple abelian variety $B$ with $\mathrm{CM}$ by a subfield $L$ of $K$ of degree $[L: \mathbb{Q}]=\frac{1}{m}[K: \mathbb{Q}]$. The CM type of $A$ arises from that of $B$ by extending the embeddings of $L$ to $K$. Therefore symmetries of the CM type of $A$ show whether $A$ is simple or not.

An abelian variety $T$ is called of $C M$ type if it is isogenous to a direct product of factors with complex multiplication. The corresponding points in a Shimura variety are called CM points or special points. In the easiest example where the upper half plane $\mathcal{H}$ parametrizes the family of all elliptic curves, the imaginary quadratic points give the CM points if we pass to the Shimura variety $\Gamma \backslash \mathcal{H}, \Gamma$ denoting the elliptic modular group.

\subsection{The splitting pattern of the Pryms}

We come back to the Prym varieties defined in Section 1.2 and collect results of [21, Satz 4] and [2, Exemple 3, Thm. 1, Lemme 1].

Proposition 2.4 Let $C$ be the subalgebra of $\operatorname{End}_{0} T(k, z)$ of elements commuting with $\mathbb{Q}\left(\zeta_{k}\right) \subseteq$ End $_{0} T(k, z)$. This subalgebra belongs to one of the following three types.

1. $C=\mathbb{Q}\left(\zeta_{k}\right)$. Then $T(k, z)$ is isogenous to a power $D^{m}$ of a simple abelian variety $D$ whose endomorphism algebra $S$ is a subfield $S \subseteq$ $\mathbb{Q}\left(\zeta_{k}\right)$ with

$$
m=\left[\mathbb{Q}\left(\zeta_{k}\right): S\right] \quad \text { and } \quad \operatorname{dim} D=[S: \mathbb{Q}]
$$

In particular, no simple factor of $T(k, z)$ has complex multiplication.

2. $C=K$ is a quadratic extension of $\mathbb{Q}\left(\zeta_{k}\right)$. The Prym variety has complex multiplication by $K$ and is isogenous to a power $B^{m}$ of a simple abelian variety with $C M$ by a subfield $L \subseteq K$ with $m=[K: L]$.

3. $C$ has zero divisors. Then $T(k, z)$ is isogenous to $A_{1} \oplus A_{2}$ with two abelian varieties $A_{i}$ of dimension $\frac{1}{2} \varphi(k)$ and with endomorphism algebra End $_{0} A_{i} \subseteq \mathbb{Q}\left(\zeta_{k}\right)$. Both $A_{i}$ have complex multiplication by $\mathbb{Q}\left(\zeta_{k}\right)$.

The proof can be sketched as follows. If $C$ has a zero divisor, its image of $T(k, z)$ gives a proper $\mathbb{Q}\left(\zeta_{k}\right)$-invariant abelian subvariety $A_{1}$ and a $\mathbb{Q}\left(\zeta_{k}\right)$ invariant complement $A_{2}$. It is well known that for such abelian varieties 
$\left[\mathbb{Q}\left(\zeta_{k}\right): \mathbb{Q}\right]$ divides $2 \operatorname{dim} A_{i}$, therefore we have equality, hence CM - the third case of the classification.

If $C$ has no zero divisors, it is a (commutative) field by [2, Lemme 1] and by reasons of divisibility again, it is either $\mathbb{Q}\left(\zeta_{k}\right)$ or a quadratic extension of it. If $C=\mathbb{Q}\left(\zeta_{k}\right),[2$, Exemple 3] applies to give the first case of our classification. The second case is now obvious by the information given in the last subsection.

\subsection{Pryms not of CM type}

Now we suppose $z=\tau \in \overline{\mathbb{Q}}$ and consider all eigenspaces $V_{n}$ as vector spaces over $\overline{\mathbb{Q}}$. Proposition 2.2 implies in particular

Lemma 2.5 Suppose $\tau \in \overline{\mathbb{Q}}, \neq 0,1$, and suppose that $T(k, \tau)$ is an abelian variety not of CM type, see Proposition 2.4.1. Then all periods

$$
\int_{\gamma} \eta, \quad \gamma \in H_{1}(T(k, \tau), \mathbb{Z})
$$

of a fixed nonzero $\eta \in V_{n} \subset H_{D R}^{1}(T(k, \tau))$ generate a $\overline{\mathbb{Q}}$-vector space $\Pi_{\eta}$ of dimension 2 .

The upper bound $\leq 2$ for this dimension follows directly from the facts that $H_{1}(T(k, \tau), \mathbb{Z})$ is a $\mathbb{Z}\left[\zeta_{k}\right]$-module of rank 2 and that $\eta$ is an eigendifferential. On the other hand, dimension $=1$ would lead to a contradiction as follows. Recall that by Proposition 2.4.1, $T(k, z)$ has only one simple factor $D$ of dimension $g=\varphi(k) / m$ and with End $_{0} D=S, S$ a number field of degree $g$. Complete $\eta$ to a basis of $H_{D R}(D)$ consisting of $2 g$ eigendifferentials for the action of $\mathbb{Q}\left(\zeta_{k}\right)$. As $\eta$, all of them have their periods in an at most 2 -dimensional $\overline{\mathbb{Q}}$-vector space. On the other hand, this upper bound is attained because Proposition 2.2 shows that all periods on $T(k, z)$ together with 1 and $\pi$ generate a $\overline{\mathbb{Q}}$-vector space of dimension $2+4 g^{1}$.

\subsection{Pryms of CM type}

Next we consider case 2 of Proposition 2.4.

\footnotetext{
${ }^{1}$ In [15, Prop. 4.1] we treated only the case that $T(k, z)$ is simple without CM, i.e. we overlooked the possibility that it can be isogenous to $D^{m}$ with $m>1$ as described in Proposition 2.4.1. However, the result remains true (see Lemma 2.5) also in the nonsimple case, so all consequences drawn in [15] are correct.
} 
Lemma 2.6 Suppose $\tau \in \overline{\mathbb{Q}}, \neq 0,1$ and suppose that $T(k, \tau)$ has complex multiplication by a $C M$ field $K,\left[K: \mathbb{Q}\left(\zeta_{k}\right)\right]=2$. All periods

$$
\int_{\gamma} \eta, \quad \gamma \in H_{1}(T(k, \tau), \mathbb{Z})
$$

of a nonzero second kind $\mathbb{Q}\left(\zeta_{k}\right)$-eigendifferential $\eta \in V_{n} \subset H_{D R}^{1}(T(k, \tau))$ generate a $\overline{\mathbb{Q}}$-vector space $\Pi_{\eta}$ of dimension

- 1 if $\eta$ is a $K$-eigendifferential,

- 2 if not.

The first case happens in precisely two onedimensional subspaces of $V_{n}$.

For the proof recall that $T(k, \tau)$ is isogenous to a power $B^{m}$ of a simple abelian variety with complex multiplication by some subfield $L$ of $K$, and $V_{n}$ splits into two $L$-eigenspaces for factors $B$ but for different eigenvalues. Then the result follows again from Proposition 2.2.

The last possibility is case 3 of Proposition 2.4.

Lemma 2.7 Suppose $\tau \in \overline{\mathbb{Q}}, \neq 0,1$ and that $T(k, \tau)$ is isogenous to $A_{1} \oplus$ $A_{2}$ for two abelian varieties of $A_{i}$ dimension $\frac{1}{2} \varphi(k)$ and with complex multiplication by $\mathbb{Q}\left(\zeta_{k}\right)$.

1. If $A_{1}$ and $A_{2}$ have the same CM type, all periods

$$
\int_{\gamma} \eta, \quad \gamma \in H_{1}(T(k, \tau), \mathbb{Z}), \eta \in V_{n}
$$

of any eigenspace $V_{n} \subset H_{D R}^{1}(T(k, \tau))$ generate a $\overline{\mathbb{Q}}$-vector space $\Pi_{n}$ of dimension 1 , and $\Pi_{n}=\Pi_{\eta}$ for all nonzero $\eta \in V_{n}$.

2. If $A_{1}$ and $A_{2}$ have different $C M$ types, we have $\operatorname{dim} \Pi_{n}=2$, and the periods of every fixed $0 \neq \eta \in V_{n}$ generate a 2 -dimensional vector space $\Pi_{\eta}$ over $\overline{\mathbb{Q}}$, except in the case that $\eta$ belongs to one of the factors in the decomposition

$$
H_{D R}^{1}(T(k, \tau))=H_{D R}^{1}\left(k, A_{1}\right) \oplus H_{D R}^{1}\left(k, A_{2}\right) .
$$

In this case (happening in precisely two onedimensional subspaces of $\left.V_{n}\right) \Pi_{\eta}$ is of dimension 1 . 
In both cases the $A_{i}$ are isogenous to pure powers $B_{i}^{m_{i}}$ of simple abelian varieties $B_{i}$ with complex multiplication. In the first case, $B_{1}$ and $B_{2}$ are isogenous, and in the second case not. Then the result follows again by Wüstholz' analytic subgroup theorem in the version of Proposition 2.2, similar to Lemma 2.6. For more details the reader my consult also the proof of [15, Prop. 4.4]. Finally we give precise conditions under which the first case of Lemma 2.7 occurs. Note that these conditions do not depend on the algebraicity of $z$.

Proposition 2.8 The following statements are equivalent.

- The Shimura family $\mathcal{A}$ in Proposition 1.2 has dimension $r=0$.

- For one (hence for all) $z \neq 0,1$, the CM type of $T(k, z)$ satisfies

$$
r_{n}=0 \text { or } 2 \quad \text { for all } n \in(\mathbb{Z} / k \mathbb{Z})^{*} \text {. }
$$

- For one (hence for all) $z \neq 0,1$, the abelian variety $T(k, z)$ is isogenous to $A_{1} \oplus A_{2}$, both $A_{i}$ have dimension $\frac{1}{2} \varphi(k)$ and complex multiplication by $\mathbb{Q}\left(\zeta_{k}\right)$ with equal CM type.

- The monodromy group of the corresponding hypergeometric differential equation is finite.

- The corresponding triangle function $D\left(\nu_{0}, \nu_{1}, \nu_{\infty} ; z\right)$ is an algebraic function of $z$.

The equivalence between the first and the second point follows from Proposition 1.2. The equivalence between the second and the third is known by work of Shimura [17, Thm. 5, Prop. 14], the equivalence between the first and the last two points has been discussed already in Subsection 1.4. The equivalence between the last two points is classical, of course, see e.g. $[10, \S 57]$.

\section{Special values of Schwarz triangle functions}

\subsection{The role of complex multiplication}

We work still under the hypothesis $z=\tau \in \overline{\mathbb{Q}}$ and recall that the cycles $\gamma_{1}, \gamma_{2}$ in the definition $D\left(\nu_{0}, \nu_{1}, \nu_{\infty} ; \tau\right)=D(\tau)=\int_{\gamma_{1}} \eta(\tau) / \int_{\gamma_{2}} \eta(\tau)$ are generators of the 2 -dimensional $\mathbb{Q}\left(\zeta_{k}\right)$-module $H_{1}(T(k, \tau), \mathbb{Q})=\mathbb{Q} \otimes_{\mathbb{Z}} H_{1}(T(k, \tau), \mathbb{Z})$. Numerator and denominator generate the period vector space $\Pi_{\eta}$ discussed in the last section. We conclude from Lemmata $2.5,2.6$ and 2.7 
Theorem 3.1 Suppose $\tau \in \overline{\mathbb{Q}}, \neq 0,1$.

$$
D\left(\nu_{0}, \nu_{1}, \nu_{\infty} ; \tau\right)=D(\tau)=\frac{\int_{\gamma_{1}} \eta(\tau)}{\int_{\gamma_{2}} \eta(\tau)}
$$

is algebraic or $\infty$ if and only if $T(k, \tau)$ is of CM type and $\operatorname{dim}_{\overline{\mathbb{Q}}} \Pi_{\eta(\tau)}=1$, i.e. if $\eta(\tau)$ is a

- K-eigendifferential under the hypotheses of Proposition 2.4.2, or a

- $\mathbb{Q}\left(\zeta_{k}\right)$-eigendifferential on one of the factors $A_{1}, A_{2}$ under the hypotheses of Proposition 2.4.3.

In two special situations we can give more explicit conditions. The first is obvious by Proposition 2.8 and Lemma 2.7.1

Theorem 3.2 If the monodromy group $\Delta$ of the corresponding differential equation is finite, all values $D(\tau)$ of the triangle function at algebraic arguments $\tau$ are algebraic or $\infty$.

In the following we will therefore restrict our attention to infinite monodromy groups $\Delta$. In these cases, we know by Proposition 2.8 that at least one $r_{n}=1$, in other words one $W_{n}=V_{n} \cap H^{0}(T(k, \tau), \Omega)$ contains a nonzero differential $\eta=\omega$ of the first kind, unique up to multiples. For periods of the first kind we can apply a sharper version of Wüstholz' theorem giving a period vector space $\Pi_{\omega}$ of dimension 1 if the abelian variety has CM type. Another way to prove $\operatorname{dim} \Pi_{\omega}=1$ is a second look on Lemma 2.6 and Lemma 2.7: in Lemma 2.6, $H^{0}(T(k, \tau), \Omega)$ is $K$-invariant, therefore $W_{n}$ is one of the onedimensional subspaces of $K$-eigendifferentials. In Lemma 2.7, $W_{n}$ belongs to precisely one of the homology factors $H^{0}\left(A_{i}, \Omega\right)$ since only one of them contains eigendifferentials $\omega$ with $\omega \circ \sigma=\zeta_{k}^{n} \omega$, otherwise we would have $\operatorname{dim} W_{n}=2$. Summing up we get (see also [16, Cor. 5] for a different argument)

Theorem 3.3 Suppose $\tau \in \overline{\mathbb{Q}} \neq 0,1$, and that $T(k, \tau)$ is of CM type, let $W_{n}$ be a one-dimensional $\mathbb{Q}\left(\zeta_{k}\right)$-eigenspace in $H^{0}(T(k, \tau), \Omega)$. If $0 \neq$ $\omega=\eta(\tau) \in W_{n}$, the value of the corresponding triangle function $D(\tau)=$ $\int_{\gamma_{1}} \eta(\tau) / \int_{\gamma_{2}} \eta(\tau)$ is algebraic.

The first natural question is now: how to control that $\eta$ is of first kind? For simplicity, take $n=1$. There $\eta=d u / y$ - see Section $1.1-$ is of first kind if and only if the exponential parameters $\mu_{j}$ are all $<1$. The second 
question is already much more difficult: for which $\tau \in \overline{\mathbb{Q}}$ is $T(k, \tau)$ of $\mathrm{CM}$ type? The answer depends on the nature of the monodromy group $\Delta$ and, unfortunately, does not give a general explicit criterion for the distinction between $\mathrm{CM}$ and non-CM cases.

1. If $\Delta$ is finite, $T(k, z)$ is of CM type for every $z$, see Proposition 2.8 .

2. If $\Delta$ is an arithmetic group, there is an infinity of $T(k, \tau)$ of CM type and an infinity of $T(k, \tau)$ not of CM type. In these cases - classified by Takeuchi [19] $-\Delta$ is commensurable to the modular group for a complex onedimensional family of polarized abelian varieties with a certain endomorphism structure. Our $T(k, z), z \neq 0,1$, form a dense subset of this family, and the Schwarz triangle function $D$ is the inverse function of an arithmetic automorphic function for this modular group, possibly up to composition with an algebraic function. See also our remarks about Shimura curves in Section 1.4 and about CM points in Section 2.3.

3. If $\Delta$ is infinite and non-arithmetic, the $T(k, z)$ form a subfamily not of Hodge type in the Shimura variety of all polarized abelian varieties of their endomorphism structure. In this case, the André-Oort conjecture predicts that there are only finitely many $T(k, \tau)$ of CM type. This conjecture is proven by Edixhoven and Yafeev [7] for those CM types discussed in Proposition 2.4.3, but it is open in general. For more information and applications to other hypergeometric questions see $[6]$.

\subsection{Other algebraic values at algebraic arguments}

The aim of this part is to show that Theorems 3.2 and 3.3 describe very exceptional situations, i.e. that in general for $\tau \in \overline{\mathbb{Q}}-\{0,1\}$

$$
D(\nu ; \tau)=D\left(\nu_{0}, \nu_{1}, \nu_{\infty} ; \tau\right) \notin \overline{\mathbb{Q}}
$$

even if the necessary condition given by Theorem 3.1 is satisfied that $T(k, \tau)$ is of CM type. We used here an abbreviated notation $\nu:=\left(\nu_{0}, \nu_{1}, \nu_{\infty}\right)$ for the rational triplets of angular parameters (always under the restriction (1.1)). We call two such triplets $\nu, \nu^{\prime}$ associate if they belong to associate hypergeometric functions, see the conditions on their components given in Section 1.3. Observe that triangle functions with associate angular parameters belong to the same monodromy group. 
Theorem 3.4 Let $P$ be a finite set of associate rational angular parameter triplets $\nu$, belonging to an infinite monodromy group $\Delta$. There is a finite set $E_{P} \subset \overline{\mathbb{Q}}$ of exceptional arguments such that for all other $\tau \in \overline{\mathbb{Q}}-E_{P}$ at most two of the values $D(\nu ; \tau), \nu \in P$, are algebraic or $\infty$.

We may assume that $P$ contains more than two elements and that $0,1 \in$ $E_{P}$, and for the proof we may assume moreover that $T(k, \tau)$ is of $\mathrm{CM}$ type since we know by Theorem 3.1 that otherwise all values in question are transcendental. Theorem 3.4 uses Lemma 1.1 and classical facts about associate hypergeometric functions: denote the differentials in the integral representation of $D(\nu ; z), \nu \in P$, by $\eta(\nu ; z)$ and observe that all these $\eta(\nu ; z), \nu \in P$, belong to one eigenspace $V_{n}$. By Gauss' relations among contiguous hypergeometric functions, any two of them generate $V_{n}$ as a $\mathbb{C}(z)$-vector space. The only obstacle is mentioned already in Section 1.3 that for a fixed value $z=\tau$ they may fail to be a basis over $\mathbb{C}$ or $\overline{\mathbb{Q}}$. A closer look into Gauss' relations [8] shows that this can happen only at finitely many algebraic points since the relations always have coefficients in $\mathbb{Q}(z)$ : For any three different fixed associate $\eta(\nu ; z), \eta\left(\nu^{\prime} ; z\right), \eta\left(\nu^{\prime \prime} ; z\right)$ we get a representation

$$
\eta(\nu ; z)=r^{\prime}(z) \eta\left(\nu^{\prime} ; z\right)+r^{\prime \prime}(z) \eta\left(\nu^{\prime \prime} ; z\right)
$$

with nonvanishing rational functions $r^{\prime}, r^{\prime \prime} \in \mathbb{Q}(z)$. We can use these relations in all special points $\tau \in \overline{\mathbb{Q}}$ as relations over $\overline{\mathbb{Q}}$, except for the (algebraic) poles of $r^{\prime}, r^{\prime \prime}$. For $\nu, \nu^{\prime}, \nu^{\prime \prime} \in P$ we obtain finitely many such poles and also finitely many algebraic zeros of all such $r, r^{\prime}$. If we include these finitely many exceptions in our exceptional set $E_{P}$, in all other points $\tau \in \overline{\mathbb{Q}}$ the $\eta(\nu, \tau), \nu \in P$, generate pairwise different one-dimensional subspaces of $V_{n}$. Lemma 2.6 and Lemma 2.7.2 show that in only two such one-dimensional subspaces the period vector spaces $\Pi_{\eta}$ are of dimension 1 , and this is equivalent to the algebraicity of the period quotient $D(\nu ; \tau)$.

It seems to be very likely that $E_{P} \supseteq\{0,1\}$ is finite even for infinite sets $P$ of associate parameter triplets because in exceptional points $\tau \neq 0,1$ three quite different conditions have to be satisfied. First,

- two $\eta(\nu ; \tau), \eta\left(\nu^{\prime} ; \tau\right), \nu, \nu^{\prime} \in P$, have to be multiples of each other.

As an example that this can happen take relation (28) on p. 103 of [8]

$$
\begin{aligned}
(c-a) F(a-1, b, c ; z) & +(2 a-c-a z+b z) F(a, b, c ; z) \\
& +a(z-1) F(a+1, b, c ; z)=0 .
\end{aligned}
$$


Translated to the language of differentials and angular parameters it says that in the special point $\tau=(2 a-c) /(a-b)=\left(\nu_{\infty}-\nu_{1}\right) / \nu_{\infty}$ the associate differentials

$$
\eta\left(\nu_{0}, \nu_{1}+1, \nu_{\infty}+1 ; \tau\right), \quad \eta\left(\nu_{0}, \nu_{1}-1, \nu_{\infty}-1 ; \tau\right)
$$

are $\overline{\mathbb{Q}}$-linearly dependent and give there the same period quotient $D(\tau)$. Whether or not this value is really algebraic depends of course on two further conditions, namely

- if $T(k, \tau)$ is of CM type and

- if $\eta(\nu ; \tau)$ generates one of the two one-dimensional eigenspaces mentioned in Lemma 2.6 or Lemma 2.7.2.

In general, the second and the third condition are difficult to verify, for examples see the next Sections. In the case treated in Theorem 3.3 we can better localize at least one of these one-dimensional eigenspaces: it is the subspace of differentials of the first kind but containing no other $\eta(\nu ; \tau)$, if we have no coincidences coming from the degeneration of Gauss' relations discussed in the proof of Theorem 3.4. Therefore we get the following sharper result.

Theorem 3.5 Let $P$ be a finite set of associate rational angular parameter triplets $\nu$, belonging to an infinite monodromy group $\Delta$, and suppose further that there is precisely one first kind differential $\omega=\eta\left(\nu^{\prime} ; z\right)$ associate to these $\eta(\nu ; z), \nu \in P$, but with $\nu^{\prime} \notin P$. Then there is a finite set $E_{P} \subset \overline{\mathbb{Q}}$ of exceptional arguments such that for all $\tau \in \overline{\mathbb{Q}}-E_{P}$ at most one of the values $D(\nu ; \tau), \nu \in P$, is algebraic or $\infty$.

\section{Examples of algebraic values: Pryms of CM type}

As for the special values of the Schwarz map $D(z)$ for a differential $\eta(z)$ on a family of hypergeometric curves, we have established the general properties in preceding sections. Here we consider examples explaining the situation in question. They all arise from specializations of a family of curves studied in the framework of ball quotients and Appell-Lauricella hypergeometric functions in two variables. 


\subsection{Pentagonal curves and their degeneration}

Let us consider a family of hypergeometric curves given by (1.2):

$$
\begin{gathered}
X(p, z)=X(z): \\
y^{p}=x^{p \mu_{0}}(x-1)^{p \mu_{1}}(x-z)^{p \mu_{z}} \quad(z \in \mathbb{C}-\{0,1\}),
\end{gathered}
$$

where we suppose $p$ to be a prime and $\mu_{0}, \mu_{1}, \mu_{z}, \mu_{\infty} \notin \frac{1}{2} \mathbb{Z}$, equivalent to the non-integrality condition (1.1). We defined the Prym variety $T(p, z)$ for $X(z)$ induced from the Jacobi variety $\operatorname{Jac}(X(z))$. Since $k=p$ is prime, $T(p, z)$ coincides with $\operatorname{Jac}(X(z))$. So in our case the field $\mathbb{Q}\left(\zeta_{p}\right)$ acts on the space of holomorphic differentials $H^{0}(\operatorname{Jac}(X(z)), \Omega) \cong H^{0}(X(z), \Omega)$ with parameter $z$. We note also that the $\mathbb{Q}\left(\zeta_{p}\right)$-action on $X(z)$ induces a $\mathbb{Q}\left(\zeta_{p}\right)$ module structure on $H_{1}(X(z), \mathbb{Q})$ of rank two. Let $\gamma_{1}, \gamma_{2}$ be two 1-cycles on $X(z)$ independent over $\mathbb{Q}\left(\zeta_{p}\right)$ and let as in Subsection 1.1

$$
\eta(z)=x^{-\mu_{0}}(x-1)^{-\mu_{1}}(x-z)^{-\mu_{z}} d x
$$

be a differential of second kind on $X(z)$. Then the corresponding Schwarz map is defined by (1.3). Let $P\left(\lambda_{1}, \lambda_{2}\right)$ be a projective nonsingular model of the affine curve

$$
y^{5}=x(x-1)\left(x-\lambda_{1}\right)\left(x-\lambda_{2}\right), \quad\left(\lambda_{1}, \lambda_{2}, \lambda_{1} / \lambda_{2}, \in \mathbb{C}-\{0,1\}\right) .
$$

$P\left(\lambda_{1}, \lambda_{2}\right)$ is a curve of genus 6 and is called a pentagonal curve. There are many articles concerned with this family. We cite here just one by K. Koike [12]. We have a basis of $H^{0}\left(P\left(\lambda_{1}, \lambda_{2}\right), \Omega\right)$ :

$$
\varphi_{1}=\frac{d x}{y^{2}}, \varphi_{2}=\frac{d x}{y^{3}}, \varphi_{3}=\frac{x d x}{y^{3}}, \varphi_{4}=\frac{d x}{y^{4}}, \varphi_{5}=\frac{x d x}{y^{4}}, \varphi_{6}=\frac{x^{2} d x}{y^{4}} .
$$

Let $\operatorname{Deg} P(z)$ be the compact nonsingular model of

$$
y^{5}=x^{2}(x-1)(x-z) \quad(z \in \mathbb{C}-\{0,1\}) .
$$

It is a degenerate pentagonal curve of genus 4 . There is a natural $\zeta_{5}$-action

$$
\sigma:(x, y) \mapsto\left(x, \zeta_{5}^{-1} y\right) .
$$

So we have

$$
\mathbb{Q}\left(\zeta_{5}\right) \subseteq \operatorname{End}_{0}(\operatorname{Jac}(\operatorname{Deg} P(z)))
$$


We have a basis of $H^{0}(\operatorname{Deg} P(z), \Omega)$ :

$$
\omega_{1}=\frac{d x}{y^{2}}, \omega_{2}=\frac{x d x}{y^{3}}, \omega_{3}=\frac{x d x}{y^{4}}, \omega_{4}=\frac{x^{2} d x}{y^{4}}
$$

consisting of eigendifferentials for the action of $\mathbb{Q}\left(\zeta_{5}\right)$.

Remark 4.1 We note that $\omega_{3}$ and $\omega_{4}$ are mutually associate.

In general we have a solution for the Gauss hypergeometric differential equation

$$
E(a, b, c) \quad: \quad z(1-z) f^{\prime \prime}+(c-(1+a+b) z) f^{\prime}-a b f=0
$$

given by the integrals

$$
\begin{aligned}
& e^{-\pi i(-c+b+1-a)} \int_{1}^{\infty} x^{a-c}(x-1)^{c-b-1}(x-z)^{-a} d x \\
= & e^{-\pi i(-c+b+1-a)} \int_{0}^{1} u^{b-1}(1-u)^{c-b-1}(1-z u)^{-a} d u \\
= & \int_{1}^{\infty} x^{a-c}(1-x)^{c-b-1}(z-x)^{-a} d x=F_{1 \infty}(a, b, c ; z)
\end{aligned}
$$

with

$$
u x=1,1-x=e^{-\pi i}(x-1), z-x=e^{\pi i}(x-z) .
$$

That solution is single valued holomorphic at $z=0$ and

$$
F(a, b, c ; z)=e^{\pi i(1-c+b-a)} \frac{\Gamma(c)}{\Gamma(b) \Gamma(c-b)} F_{1 \infty}(a, b, c ; x) .
$$

Then the integral

$$
\int_{1}^{\infty} \omega_{1}(z)
$$

is a holomorphic solution of $E(2 / 5,3 / 5,6 / 5)$ at $z=0$. The absolute values of the angular parameters are given by

$$
(|1-c|,|c-a-b|,|a-b|)=\left(\frac{1}{5}, \frac{1}{5}, \frac{1}{5}\right) .
$$

By putting $x=1 / x_{1}, y=z^{1 / 5} y_{1} / x_{1}, z=1 / z_{1}$ in $(4.2)$ we obtain an isomorphic nonsingular curve given by

$$
y_{1}^{5}=x_{1}\left(x_{1}-1\right)\left(x_{1}-z_{1}\right),
$$


the integral $\int d x_{1} / y_{1}^{2}$ gives a solution for $E(2 / 5,1 / 5,4 / 5)$ with the same angular parameters in absolute values. So the inverse of the Schwarz map becomes an automorphic function on the upper half plane with respect to a cocompact arithmetic triangle group $\Delta(5,5,5)$. An explicit expression of this automorphic function is given by Koike [12, Theorem 6.3].

\subsection{First example}

Theorem 4.2 In the family of curves (4.2), $T\left(5,-\zeta_{3}\right)$ has complex multiplication by the field $\mathbb{Q}\left(\zeta_{15}\right)$. For the differentials (4.3), the value of the Schwarz map $D\left(\omega_{3},-\zeta_{3}\right)=D\left(-\frac{2}{5},-\frac{3}{5},-\frac{2}{5} ;-\zeta_{3}\right)$ is transcendental, but

$$
\begin{gathered}
D\left(\omega_{1},-\zeta_{3}\right)=D\left(-\frac{1}{5}, \frac{1}{5},-\frac{1}{5} ;-\zeta_{3}\right) \\
D\left(\omega_{2},-\zeta_{3}\right)=D\left(\frac{1}{5},-\frac{1}{5}, \frac{1}{5} ;-\zeta_{3}\right) \\
D\left(\omega_{4},-\zeta_{3}\right)=D\left(\frac{3}{5},-\frac{3}{5}, \frac{3}{5} ;-\zeta_{3}\right)
\end{gathered}
$$

are algebraic numbers.

Proof. Define

$$
\Sigma: w^{5}=t^{2}\left(t^{3}-1\right) .
$$

It is a singular model of a curve of genus 4 and we have on this model a basis of the space of holomorphic differentials:

$$
\varphi_{1}=\frac{d t}{w^{2}}, \varphi_{2}=\frac{t d t}{w^{3}}, \varphi_{3}=\frac{t d t}{w^{4}}, \varphi_{4}=\frac{t^{2} d t}{w^{4}} .
$$

There are actions of $\zeta_{3}$ and $\zeta_{5}$ :

$$
\begin{gathered}
t^{\prime}=\zeta_{3} t, w^{\prime}=\zeta_{3} w \\
t^{\prime}=t, w^{\prime}=\zeta_{5} w
\end{gathered}
$$

on $\Sigma$. They generate a cyclic group of automorphisms on $\Sigma$ generated by a single action

$$
t^{\prime}=\zeta_{15}^{5} t, w^{\prime}=\zeta_{15}^{2} w
$$


and induces an action of $\mathbb{Q}\left(\zeta_{15}\right)$ on the space of holomorphic differentials. Any $\varphi_{i}(i=1,2,3,4)$ is an eigendifferential for this action, and $\mathbb{Q}\left(\zeta_{15}\right)$ acts faithfully on the space of holomorphic differentials. We have

$$
\left[\mathbb{Q}\left(\zeta_{15}\right): \mathbb{Q}\right]=8=2 \cdot \text { genus of } \Sigma .
$$

It means $\operatorname{End}_{0} \operatorname{Jac}(\Sigma)=\mathbb{Q}\left(\zeta_{15}\right)$ and that $\operatorname{Jac}(\Sigma)$ is an abelian variety with complex multiplication by $\mathbb{Q}\left(\zeta_{15}\right)$. A more detailed analysis of its CM type shows that it is simple and that the $\varphi_{i}$ generate the eigenspaces in $H^{0}(\Sigma, \Omega)$.

Defining

$$
T: t(x)=\frac{x}{\zeta_{3}\left(-1+\zeta_{3}+x\right)}, \quad w(x, y)=\frac{(-1)^{\frac{1}{10}} 3^{\frac{3}{10}} y}{-1+\zeta_{3}+x},
$$

the CM curve $\Sigma$ is transformed to the degenerated pentagonal hypergeometric curve

$$
\operatorname{Deg} P\left(-\zeta_{3}\right): y^{5}=x^{2}(x-1)\left(x+\zeta_{3}\right)
$$

whose Prym variety $T\left(5,-\zeta_{3}\right)$ (here it is just the Jacobian) belongs therefore to those discussed in case 2 of Proposition 2.4.

The converse transformation $T^{-1}$ is given by

$$
x(t)=\frac{\left(1-\zeta_{3}\right) t}{-\zeta_{3}^{2}+t}, y(t, w)=\frac{3^{\frac{1}{5}}\left(\zeta_{3}\right)^{\frac{1}{5}} w}{-1+\zeta_{3} t} .
$$

The pullback of the differentials under the transformation $T$ is

$$
\begin{array}{r}
T^{*}\left(\omega_{1}\right)=\frac{\left(-\left(\frac{1}{3}\right)\right)^{\frac{2}{5}}\left(-1+(-1)^{\frac{2}{3}}\right)}{w^{2}} d t \\
T^{*}\left(\omega_{2}\right)=\frac{(-1)^{\frac{23}{30}}\left(-1+(-1)^{\frac{2}{3}}\right) t}{3^{\frac{1}{10}} w^{3}} d t \\
T^{*}\left(\omega_{3}\right)=\frac{(-1)^{\frac{19}{30}}\left(-1+(-1)^{\frac{2}{3}}\right) t\left(-1+(-1)^{\frac{2}{3}} t\right)}{3^{\frac{3}{10}} w^{4}} d t \\
T^{*}\left(\omega_{4}\right)=-\left(\frac{(-1)^{\frac{2}{15}} 3^{\frac{1}{5}}\left(-1+(-1)^{\frac{2}{3}}\right) t^{2}}{w^{4}}\right) d t .
\end{array}
$$

So via the transformation $T, \omega_{1}, \omega_{2}$ and $\omega_{4}$ are equal to $\varphi_{1}, \varphi_{2}$ and $\varphi_{4}$ up to a constant factor, respectively. But $\omega_{3}$ is a linear combination of $\varphi_{3}$ and $\varphi_{4}$ and it is not an eigendifferential for the action of the CM field $\mathbb{Q}\left(\zeta_{15}\right)$. 
If we consider the Schwarz map

$$
D\left(\omega_{j}, z\right)=\frac{\int_{\gamma_{1}} \omega_{j}}{\int_{\gamma_{2}} \omega_{j}} \quad(j=1,2,3,4)
$$

for the family $\{\operatorname{Deg} P(z)\}$ with respect to the differentials $\omega_{j}$, Theorem 4.2 follows directly from Theorem 3.1 and Lemma 2.6.

\subsection{Second example}

Now we study the same family of curves at the point $\tau=-1$

$$
\operatorname{Deg} P(-1)=\Sigma^{\prime}: y^{5}=x^{2}\left(x^{2}-1\right)
$$

and show that its Jacobian belongs to those studied in case 3 of Proposition 2.4 .

\section{Theorem 4.3}

$$
\operatorname{Jac}\left(\Sigma^{\prime}\right)=T(5,-1) \quad \text { is isogenous to } A_{1} \oplus A_{2}
$$

with End ${ }_{0}\left(A_{i}\right)=\mathbb{Q}\left(\zeta_{5}\right)$. For all differentials in (4.3) the Schwarz maps have algebraic values $D\left(\omega_{i},-1\right)(i=1,2,3,4)$.

Consider

$$
\text { HypE : } y^{5}=u(u-1) \text {. }
$$

We have a natural map

$$
\Sigma^{\prime} \rightarrow \text { HypE }
$$

by $x \mapsto u=x^{2}$. It shows that $\operatorname{Jac}\left(\Sigma^{\prime}\right)$ is not simple and $A_{1}=\operatorname{Jac}(H y p E)$ is a component. The differentials

$$
\omega_{2}=\frac{x d x}{y^{3}}, \quad \omega_{3}=\frac{x d x}{y^{4}}
$$

are the lifts from those on $H y p E$. The action of $\zeta_{5}$ is given by $\sigma:(x, y) \mapsto$ $\left(x, \zeta_{5}^{-1} y\right)$. So

$$
\sigma\left(\omega_{2}\right)=\sigma\left(\frac{x d x}{y^{3}}\right)=\zeta_{5}^{3} \omega_{2}, \quad \sigma\left(\omega_{3}\right)=\sigma\left(\frac{x d x}{y^{4}}\right)=\zeta_{5}^{4} \omega_{3} .
$$


Hence $A_{1}$ is an abelian variety of CM type with the field $\mathbb{Q}\left(\zeta_{5}\right)$ and simple CM type $(3,4)$. As we see later the cofactor $A_{2}$ is of CM type $(4,2)$. By the change of a primitive 5 -th root of unity $A_{1}$ and $A_{2}$ are isogenous. We will see below by a period matrix calculation that we have even an isomorphism.

We consider the special values of the Schwarz maps $D\left(\omega_{2},-1\right)$ and $D\left(\omega_{3},-1\right)$. They are reduced to consider the periods

$$
\int_{1}^{\infty} \frac{d u}{y^{3}}, \quad \int_{0}^{1} \frac{d u}{y^{3}}
$$

and

$$
\int_{1}^{\infty} \frac{d u}{y^{4}}, \quad \int_{0}^{1} \frac{d u}{y^{4}}
$$

on the CM hyperelliptic curve $y^{5}=u(u-1)$. The differentials $d u / y^{3}$ and $d u / y^{4}$ are eigendifferentials for the action of the corresponding CM field $\mathbb{Q}\left(\zeta_{5}\right)$ on the factor $A_{1}$. According to Theorems $3.1,3.3$ and Lemma 2.7.2 the values $D\left(\omega_{2},-1\right)$ and $D\left(\omega_{3},-1\right)$ are algebraic. Theorem 3.3 shows the algebraicity of $D\left(\omega_{1},-1\right)$ as well. Only $\omega_{4}$ cannot be seen directly to be a differential on $A_{2}$.

We have to consider the following question: are the two associate differentials $\omega_{3}, \omega_{4}$ (see Remark 4.1) just those two differentials of Theorem 3.4 generating the two one-dimensional eigenspaces in $V_{4}$ needed according to Lemma 2.7 ? The answer will be "yes" by explicit calculation of the period matrix of $\Sigma^{\prime}: y^{5}=x^{2}\left(x^{2}-1\right)$. Set

$$
x=\frac{1}{x_{1}}, \quad y=-\frac{y_{1}}{x_{1}} .
$$

So we get an isomorphic curve $\Sigma_{1}: y_{1}^{5}=x_{1}\left(x_{1}^{2}-1\right)$. We have the expression of the basis $\left\{\omega_{1}, \omega_{2}, \omega_{3}, \omega_{4}\right\}$ on $\Sigma_{1}$ :

$$
\omega_{1}=-\frac{d x_{1}}{y_{1}^{2}}, \omega_{2}=\frac{d x_{1}}{y_{1}^{3}}, \omega_{3}=-\frac{x_{1} d x_{1}}{y_{1}^{4}}, \omega_{4}=-\frac{d x_{1}}{y_{1}^{4}} .
$$

Let $r, r^{\prime}$ be arcs on $\Sigma_{1}$ given by the oriented lines $[0,1],[-1,0]$ with real negative and real positive value $y_{1}$, respectively. Remember that $\sigma$ denotes the change of sheets induced by $y_{1} \mapsto \zeta_{5}^{-1} y_{1}$ and let $r^{(i)}$ be the arc 
$\sigma^{1-i} r \quad(i=1,2,3,4)$. Set $\alpha^{(i)}=r^{(i)}-r^{(i+1)}$ and $\beta^{(i)}=r^{(i)}-r^{(i+1)}$. Set

$$
M_{1}=\left(\begin{array}{cccccccc}
0 & -1 & 0 & 0 & 0 & 1 & 0 & 1 \\
0 & -1 & 0 & 0 & 0 & 1 & 0 & 0 \\
0 & -1 & 0 & 0 & 0 & 0 & 0 & 0 \\
0 & -1 & 0 & 1 & 0 & 0 & 0 & 0 \\
1 & -1 & 0 & 1 & 0 & 1 & 0 & 0 \\
0 & 0 & 0 & 0 & 1 & 0 & 0 & -1 \\
0 & -1 & 1 & 1 & 0 & 0 & 0 & 0 \\
0 & 0 & 0 & 1 & 1 & 0 & 1 & -1
\end{array}\right)
$$

Then

$$
\left(A_{2}, A_{3}, A_{5}, A_{6}, B_{2}, B_{3}, B_{5}, B_{6}\right)=\left(\alpha^{(1)}, \ldots, \alpha^{(4)}, \beta^{(1)}, \ldots, \beta^{(4)}\right) M_{1}
$$

is a homology basis of $\Sigma_{1}$ with the intersection matrix

$$
\left(\begin{array}{cccccccc}
0 & 0 & 0 & 0 & 1 & 0 & 0 & 0 \\
0 & 0 & 0 & 0 & 0 & 1 & 0 & 0 \\
0 & 0 & 0 & 0 & 0 & 0 & 1 & 0 \\
0 & 0 & 0 & 0 & 0 & 0 & 0 & 1 \\
-1 & 0 & 0 & 0 & 0 & 0 & 0 & 0 \\
0 & -1 & 0 & 0 & 0 & 0 & 0 & 0 \\
0 & 0 & -1 & 0 & 0 & 0 & 0 & 0 \\
0 & 0 & 0 & -1 & 0 & 0 & 0 & 0
\end{array}\right)
$$

These cycles $A_{2}, A_{3}, A_{5}, A_{6}, B_{2}, B_{3}, B_{5}, B_{6}$ are the same ones as those given by K. Koike in [12] on the general pentagonal curve going to the limit

$$
\lim _{\lambda \rightarrow-\infty} y_{1}^{5}=x_{1}\left(x_{1}-1\right)\left(x_{1}+1\right)\left(x_{1}+\lambda\right) .
$$

Put

$$
p_{i}=\int_{\alpha(1)} \omega_{i}, \quad q_{i}=\int_{\beta^{(1)}} \omega_{i} \quad(i=1,2,3,4)
$$

then we have

$$
q_{1}=-p_{1}, q_{2}=p_{2}, q_{3}=p_{3}, q_{4}=-p_{4} .
$$

Setting $\omega_{i}^{\prime}=\omega_{i} / p_{1} \quad(i=1,2,3,4)$, we have the period matrix of $\omega_{i}^{\prime}$ for the cycles $\left(\alpha^{(1)}, \ldots, \alpha^{(4)}, \beta^{(1)}, \ldots, \beta^{(4)}\right)$. 
The period matrix of $\Sigma_{1}: y_{1}^{5}=x_{1}\left(x_{1}^{2}-1\right)$ with respect to the basis $\left\{\omega_{1}^{\prime} \ldots, \omega_{4}^{\prime}\right\}$ of $H^{0}\left(\Sigma_{1}, \Omega\right)$ and the basis

$$
\left\{\alpha^{(1)}, \ldots, \alpha^{(4)}, \beta^{(1)}, \ldots, \beta^{(4)}\right\}
$$

of $H^{1}\left(\Sigma_{1}, \mathbb{Z}\right)$ is given by

$$
\left(\begin{array}{cccccccc}
1 & \zeta_{5}^{3} & \zeta_{5}^{1} & \zeta_{5}^{4} & -1 & -\zeta_{5}^{3} & -\zeta_{5}^{1} & -\zeta_{5}^{4} \\
1 & \zeta_{5}^{2} & \zeta_{5}^{4} & \zeta_{5}^{1} & 1 & \zeta_{5}^{2} & \zeta_{5}^{4} & \zeta_{5}^{1} \\
1 & \zeta_{5} & \zeta_{5}^{2} & \zeta_{5}^{3} & 1 & \zeta_{5} & \zeta_{5}^{2} & \zeta_{5}^{3} \\
1 & \zeta_{5} & \zeta_{5}^{2} & \zeta_{5}^{3} & -1 & -\zeta_{5} & -\zeta_{5}^{2} & -\zeta_{5}^{3}
\end{array}\right)
$$

By changing the $\mathbb{Q}$-homology basis to

$$
\left\{\alpha^{(1)}+\beta^{(1)}, \ldots, \alpha^{(4)}+\beta^{(4)}, \alpha^{(1)}-\beta^{(1)}, \ldots, \alpha^{(4)}+\beta^{(4)}\right\}
$$

we know that $\operatorname{Jac}\left(\Sigma_{1}\right)=T(5,-1)$ is isogenous to the direct sum

$$
\begin{aligned}
& \mathbb{C}^{2} /\left(\mathbb{Z}\left(\begin{array}{l}
1 \\
1
\end{array}\right)+\mathbb{Z}\left(\begin{array}{l}
\zeta_{5}^{2} \\
\zeta_{5}
\end{array}\right)+\mathbb{Z}\left(\begin{array}{l}
\zeta_{5}^{4} \\
\zeta_{5}^{2}
\end{array}\right)+\mathbb{Z}\left(\begin{array}{l}
\zeta_{5} \\
\zeta_{5}^{3}
\end{array}\right)\right)+ \\
& \mathbb{C}^{2} /\left(\mathbb{Z}\left(\begin{array}{l}
1 \\
1
\end{array}\right)+\mathbb{Z}\left(\begin{array}{l}
\zeta_{5}^{3} \\
\zeta_{5}
\end{array}\right)+\mathbb{Z}\left(\begin{array}{l}
\zeta_{5} \\
\zeta_{5}^{2}
\end{array}\right)+\mathbb{Z}\left(\begin{array}{l}
\zeta_{5}^{4} \\
\zeta_{5}^{3}
\end{array}\right)\right) .
\end{aligned}
$$

That means $\operatorname{Jac}\left(\Sigma^{\prime}\right)$ is $\mathbb{Q}$-isogenous to a direct sum of two 2-dimensional abelian varieties of CM type with the CM field $\mathbb{Q}\left(\zeta_{5}\right)$ of type $(3,4)$ and of type $(4,2)$, and these types are the same under the isomorphism $\zeta_{5} \mapsto \zeta_{5}^{3}$.

\section{Examples of algebraic values: symmetry and de- generation}

The results in Theorems 4.2 and 4.3 concerning $D\left(\omega_{1} ; \tau\right)$ and $D\left(\omega_{2} ; \tau\right)$ in the points $\tau=-\zeta_{3},-1$ are not at all surprising since they are easily proved with Theorem 3.3 provided we know that $T(5, \tau)$ is of CM type. Even if we know that fact, the results of the preceding section concerning $\omega_{3}$ and $\omega_{4}$ needed much more effort since they do not generate a one-dimensional eigenspace of holomorphic differentials $W_{n}$ (hypothesis of Theorem 3.3). For them it is quite remarkable that $\omega_{4}$ was a $K$-eigendifferential for Lemma 2.6 in the case $\tau=-\zeta_{3}$ or that even both belonged to the two factors in Lemma 2.7.2 in the case $\tau=-1$. In this section, we will shed some further light on these phenomena, extend parts of the previous results and explain why both $\tau$ in question are exceptional arguments in the sense of Theorems 3.4 and 3.5 . 
Theorem 5.1 1. Suppose $\rho=\nu_{0}=\nu_{1}=\nu_{\infty} \in \mathbb{Q}-\frac{1}{3} \mathbb{Z}$. Then $D\left(\rho, \rho, \rho ;-\zeta_{3}\right)$ is algebraic or $\infty$.

2. Suppose $\nu_{1}$ and $\rho=\nu_{0}=\nu_{\infty} \in \mathbb{Q}-\mathbb{Z}$ satisfy the non-integrality condition (1.1). Then $D\left(\nu_{0}, \rho, \rho ;-1\right)$ is algebraic or $\infty$.

\subsection{Symmetry arguments}

To prove the first statement, observe that in this case $\rho \in \mathbb{Q}-\frac{1}{3} \mathbb{Z}$ is a restatement of the non-integrality condition. The corresponding hypergeometric differential equation is invariant under the fractional linear transformation

$$
z \mapsto \frac{z-1}{z}
$$

inducing a cyclic permutation of the singularities $0,1, \infty$; fixed points are $\zeta_{6}$ and $-\zeta_{3}$. The image of the lower half plane has therefore a symmetry of order 3 , hence its vertices $D(0), D(1), D(\infty)$ under the Schwarz map $D$ have a midpoint $D\left(-\zeta_{3}\right)$, i.e. a fixed point of an order $3 \mathrm{PSL}_{2}(\mathbb{C})$ transformation $\mu$ providing an automorphism of the $D$-image and a cyclic permutation of the vertices. (To see that it is really a fractional linear transformation, observe that $\mu$ extends to either a disc or the Riemann sphere if one considers all analytic continuations of $D$.) These vertices are algebraic or $\infty$ what can be seen either by direct calculation as in $[21,(15)]$ or by the fact that in these points the Prym varieties $T(k, z)$ degenerate to abelian varieties of dimension $\frac{1}{2} \varphi(k)$ with complex multiplication by $\mathbb{Q}\left(\zeta_{k}\right)$. Therefore the midpoint also has to be algebraic or $\infty$. The same argument works also for $\zeta_{6}$, but the analytic continuation of $D$ to the upper half plane changes at least one of the three vertices.

The second statement can be proved similarly but with the anticonformal transformation

$$
z \mapsto \overline{z^{-1}}
$$

exchanging 0 and $\infty$ and fixing the unit circle, in particular the point -1 which can be considered as the midpoint of the border edge $D(] \infty, 0[)$. The triangle function $D$ maps the unit circle to a symmetry axis of the $D$-images of upper and lower half plane and again the algebraicity of the vertices implies $D(-1) \in \overline{\mathbb{Q}} \cup\{\infty\}$.

Another version of these symmetry arguments has been indicated for the special case $\rho=\frac{1}{5}$ already in the end of Subsection 1.4: by nonlinear relations, $D$ is related to other triangle functions for the parameter triplets 
$\frac{1}{2}, \frac{1}{3}, \frac{\rho}{2}$ (first part) or $\frac{1}{2}, \frac{\nu_{0}}{2}, \rho$ (second part). In both cases, the $D$-values in question belong to the (algebraic!) vertices of the new image triangles.

\subsection{Corollaries and Remarks}

1. By the "only if" part of Theorem 3.1, Theorem 5.1 implies that the respective Pryms $T\left(k,-\zeta_{3}\right), T(k,-1)$ are of CM type - but without the precise information given in the last section, of course.

2. The same kind of symmetry arguments as in the second part of Theorem 5.1 works for the argument $\tau=\frac{1}{2}$ if $\nu_{0}=\nu_{1}$ and for $\tau=2$ if $\nu_{1}=\nu_{\infty}$.

3. Sign changes of the angular parameters change the triangle functions at most by fractional linear transformations defined over $\overline{\mathbb{Q}}$, see $[21,(16)-$ (18)], so Theorem 5.1 covers the algebraicity results of the last section.

4. For Theorem 5.1 it does not matter whether the underlying differential is of first kind or only of second kind. For example, the parameter triplets

$$
\left(\frac{2}{5}, \frac{3}{5}, \frac{2}{5}\right) \quad, \quad\left(\frac{2}{5},-\frac{2}{5}, \frac{2}{5}\right)
$$

belong to generators of the eigenspace $V_{1}$ for the curve family (4.2), both of second kind but the algebraicity of their values are covered by Theorem 5.1.

5. As already explained in the end of subsection 3.1, we expect only finitely many $T(k, \tau)$ to be of CM type if the corresponding monodromy group is non-arithmetic. Apparently $\tau=-1, \frac{1}{2}, 2, \zeta_{6},-\zeta_{3}$ lead to these cases if suitable symmetry conditions are satisfied, independently of the arithmeticity of the monodromy group.

\subsection{Degeneration of contiguity}

As we explained in Subsection 3.2 , associate differentials $\eta(\nu ; z)$ generate one-dimensional subspaces of $V_{n}$ which are generically pairwise different. Therefore - if the monodromy group is infinite - at most two of them give algebraic values $D(\nu ; \tau)$, see Theorems 3.4 and 3.5 , if the argument $\tau$ is not an "exceptional" one where several $\eta(\nu ; \tau)$ are multiples of each other. Theorem 5.1 gives examples for such exceptional arguments because arbitrarily many associate angular parameters lead to algebraic values.

Theorem 5.2 1. Suppose $\rho \in \mathbb{Q}-\frac{1}{3} \mathbb{Z}$ and let $P$ be a set of associate angular parameter triplets

$$
(\rho+2 k, \rho+2 k, \rho+2 k), \quad k \in \mathbb{Z} .
$$


Then $\tau=\zeta_{6}$ and $-\zeta_{3}$ are exceptional arguments.

2. Suppose $\nu_{1}$ and $\rho=\nu_{0}=\nu_{\infty} \in \mathbb{Q}-\mathbb{Z}$ satisfy the non-integrality condition (1.1) and let $P$ be a set of associate parameter triplets

$$
\left(\rho+k, \nu_{1}, \rho+k\right), \quad k \in \mathbb{Z} .
$$

Then $\tau=-1$ is an exceptional argument.

The truth of Theorem 5.2 follows from Theorems 3.4 and 5.1. As we explained in the proof of Theorem 3.4, the statement implies in particular that the corresponding differentials in these exceptional points are multiples of each other. This is in turn equivalent to a degeneration of contiguity relations: generically, any two different associate differentials generate their two-dimensional eigenspace because any other can be written as a $\mathbb{C}(z)$ linear combination of them. But for a fixed argument $z=\tau$ this may fail if the coefficient functions have poles. These relations can be produced explicitely using Gauss' relations between contiguous hypergeometric functions. We illustrate this degeneration phenomenon in the second case. From the contiguity relations in $[8,(28)-(45)]$ one may deduce with Mathematica ${ }^{\mathrm{TR}}$ the relation

$$
\begin{gathered}
(1+c)(c+z(1-a+b)) F(a, b+1, c+1 ; z)= \\
c(1+c) F(a, b, c ; z)+z(1+b)(1-a+c) F(a, b+2, c+2 ; z) .
\end{gathered}
$$

In the case $\nu_{0}=\nu_{\infty}=\rho \notin \mathbb{Z}$ we have $1-a+b=c \neq-1$, and the left hand side coefficient vanishes precisely for $z=-1$. Passing to the angular parameters and to the differentials, it means that $\eta\left(\rho, \nu_{1}, \rho ;-1\right)$ and $\eta\left(\rho-2, \nu_{1}, \rho-2 ;-1\right)$ are multiples of each other. By induction, we see that for $\tau=-1$ in this family of associate differentials all elements with even $k$ are multiples of each other, and similarly all elements with $k$ odd.

An analogous argument for the first case of Theorem 5.2 should be possible, but would need explicit relations between associate hypergeometric functions

$F(a, b, c ; z), F(a+k, b+3 k, c+2 k ; z)$ and $F(a+2 k, b+6 k, c+4 k ; z)$

an extremely difficult task. In several easier cases we expect to be able to perform the calculation with computer support. 


\subsection{Gamma values}

Finally another access to Theorem 5.1 has to be mentioned. In the symmetric situations discussed here, special values of hypergeometric functions in the fixed points of the symmetries are known, see e.g. [8, (46)-(56)]. One may use them - often together with Kummer's relations between different representations of hypergeometric functions - to produce explicit formulas describing $D(-1)$ or $D\left(\zeta_{6}\right)$ in terms of products of values of the Gamma function at rational arguments. For the normalization of $D$ used in the present paper compare also [15, Thm. 5.3 and p. 649]. Take e.g. $\omega_{3}$ in (4.3); we know already by different reasons (Theorems 4.3 and 5.1) that the corresponding value of the triangle function in -1 is algebraic. Up to algebraic nonzero factors (indicated by " $~ "$ ") we can write it as

$$
D\left(-\frac{2}{5},-\frac{3}{5},-\frac{2}{5} ;-1\right) \sim \frac{\Gamma\left(\frac{4}{5}\right) \Gamma\left(\frac{1}{10}\right)}{\Gamma\left(\frac{1}{5}\right) \Gamma\left(\frac{3}{10}\right)}
$$

and verify that this quotient is algebraic. This verification can be done either explicitely using functional equation, parity relation and Gauss-Legendre's distribution relations (see e.g. [22, p. 6]; Serge Lang conjectures moreover that all algebraic relations between Gamma values at rational arguments follow from these classical relations), but this requires patience and luck. However there is an easy criterion due to Koblitz and Ogus ([11] or [22, Prop. 1]) to decide whether the algebraicity of such a product follows from classical Gamma relations. This criterion applies here and leads as well to the result; we leave it as an exercise for the reader.

\section{References}

[1] N. Archinard, Hypergeometric Abelian Varieties, Canad. J. Math. 55 (5) (2003), 897-932.

[2] D. Bertrand, Endomorphismes de groupes algébriques; applications arithmétiques, pp. 1-45 in Approximations Diophantiennes et Nombres Transcendants, Progr. Math. 31, Birkhäuser 1983.

[3] Cl. Chevalley, A. Weil, Über das Verhalten der Integrale 1. Gattung bei Automorphismen des Funktionenkörpers, Abh. Hamburger Math. Sem. 10 (1934), 358-361.

[4] P. Cohen, J. Wolfart, Modular embeddings for some non-arithmetic Fuchsian groups, Acta Arithmetica 56 (1990), 93-110 
[5] P.B. Cohen, J. Wolfart, Algebraic Appell-Lauricella Functions, Analysis 12 (1992), 359-376

[6] P.B. Cohen, G. Wüstholz, Application of the André-Oort Conjecture to some Questions in Transcendence, pp. 89-106 in A Panorama in Number Theory or The View from Baker's Garden, ed.: G. Wüstholz, Cambridge UP 2002.

[7] B. Edixhoven, A. Yafeev, Subvarieties of Shimura varieties, Ann. Math. 157 (2003), 621-645.

[8] A. Erdélyi, W. Magnus, F. Oberhettinger, F.G. Tricomi, Higher Transcendental Functions, Bateman Manuscript Project, Vol. 1. McGrawHill 1953

[9] Ph. Griffiths, J. Harris, Principles of Algebraic Geometry, Wiley 1978

[10] F. Klein, Vorlesungen über die hypergeometrische Funktion, Springer 1933.

[11] N. Koblitz, A. Ogus, Algebraicity of some products of values of the $\Gamma$ function, Proc. Symp. Pure Math. 33 (1979), 343-346

[12] K. Koike, On the family of pentagonal curves of genus 6 and associated modular forms on the ball, J.Math. Soc. Japan, 55 (2003), 165-196.

[13] S. Lang, Complex Multiplication, Springer 1983.

[14] H.A. Schwarz, Über diejenigen Fälle, in welchen die Gaußische hypergeometrische Reihe eine algebraische Funktion ihres vierten Elements darstellt, J. Reine Angew. Math. 75 (1873), 292-335

[15] H. Shiga, T. Tsutsui, J. Wolfart, Fuchsian differential equations with apparent singularities, Osaka J. Math. 41 (2004), 625-658.

[16] H. Shiga, J. Wolfart, Criteria for complex multiplication and transcendence properties for automorphic functions, J. reine angew. Math. 463 (1995), 1-25.

[17] G. Shimura, On analytic families of polarized abelian varieties and automorphic functions, Ann. Math. 78 (1963), 149-192.

[18] C.L. Siegel, Lectures on Riemann Matrices, Tata Inst., Bombay 1963 
[19] K. Takeuchi, Arithmetic triangle groups, J. Math. Soc. Japan 29 (1977), 91-106.

[20] M. Waldschmidt, Transcendance de périodes: état de connaissances, to appear in the proceedings of a conference in Mahdia 2003.

[21] J. Wolfart, Werte hypergeometrischer Funktionen, Invent. math. 92 (1988), 187-216.

[22] J. Wolfart, G. Wüstholz, Der Überlagerungsradius algebraischer Kurven und die Werte der Betafunktion an rationalen Stellen, Math. Ann. 273 (1985), 1-15

[23] G. Wüstholz, Algebraic Groups, Hodge Theory, and Transcendence, pp. 476-483 in Proc. of the ICM Berkeley 1986 (ed.: A.M. Gleason), AMS 1987

[24] G. Wüstholz, Algebraische Punkte auf analytischen Untergruppen algebraischer Gruppen, Ann. of Math. 129 (1989), 501-517. 